\title{
DETERMINAÇÃO DE RESÍDUOS DE PESTICIDAS EM PLASMA BOVINO POR CROMATOGRAFIA GASOSA- ESPECTROMETRIA DE MASSAS
}

\author{
Daniele Fernanda Maffei \\ Centro Universitário Central Paulista, 13560-000 São Carlos - SP, Brasil \\ Ana Rita de Araújo Nogueira e Silvia Helena Govoni Brondi* \\ Embrapa Pecuária Sudeste, Rodovia Washington Luiz, km 234, 13560-970 São Carlos - SP, Brasil
}

Recebido em 25/5/08; aceito em 18/3/09; publicado na web em 4/8/09

\begin{abstract}
PESTICIDES RESIDUE DETERMINATION IN CATTLE PLASMA BY GAS CHROMATOGRAPHY-MASS SPECTROMETRY.
An analytical method for the isolation based on matrix solid-phase dispersion technique and gas chromatographic determination of pesticides in cattle plasma is presented. It was fortified $0.25 \mathrm{~g}$ of plasma with pesticides and blended with $1 \mathrm{~g}$ each C18 and $\mathrm{Na}_{2} \mathrm{SO}_{4}$. The homogenized matter was transferred to a SPE cartridge, which contained $1 \mathrm{~g}$ of activated florisil with $5 \mathrm{~mL}$ acetonitrile. The analites were eluted under vaccum with $15 \mathrm{~mL}$ acetonitrile, the extract was analyzed by gas chromatography-mass spectrometry. The limit of quantification of the method was $0.04 \mathrm{mg} \mathrm{L}^{-1}$ for chlorphenvinfos and fipronil and $0.02 \mathrm{mg} \mathrm{L}^{-1}$ for cypermethrin..
\end{abstract}

Keywords: pesticides; MSPD; GC-MS.

\section{INTRODUÇÃO}

Os medicamentos veterinários são empregados na medicina veterinária para fins terapêuticos, profiláticos ou como promotores de crescimento. Seu uso inadequado, pela não observância do tempo de carência, dosagens incorretas, via de administração não recomendada, terapia indiscriminada e uso de substâncias proibidas podem deixar resíduos em produtos alimentícios de origem animal, colocando em risco a saúde humana. ${ }^{1}$ No Brasil $57 \%$ do mercado veterinário é destinado a bovinos, destacando os antimicrobianos, endoparasitas, ectoparasitas, promotores de crescimento e antibióticos. ${ }^{2}$

O Brasil detém o segundo maior rebanho bovino comercial do mundo, sendo o maior exportador mundial de carne, ${ }^{3}$ mas apresenta deficiências no controle de qualidade, tanto microbiológica, como também relacionado à presença de resíduos de substâncias químicas nos produtos de origem animal disponível no mercado. ${ }^{4} \mathrm{~A}$ presença de resíduos nos alimentos, destacando os pesticidas que são aplicados no rebanho bovino, no combate ao carrapato (Boophilus microplus) e moscados-chifres (Haematobia irritanis), podem deixar resíduos no leite e na carne comercializada, comprometendo a saúde da população se estiverem em níveis acima dos permitidos pela legislação (Food and Agriculture Organization - FAO e Organização Mundial da Saúde - OMS), podendo inibir as relações de exportação/importação entre países. ${ }^{5}$

Os métodos analíticos para monitorar resíduos de medicamentos veterinários em matrizes alimentícias, destacando os pesticidas que são utilizados no combate a ectoparasitas do rebanho bovino, devem ser rápidos, específicos e sensíveis, para permitir a detecção a baixas concentrações.

As análises de amostras de alimentos sólidas ou semissólidas, destacando as de origem biológica, são extremamente trabalhosas. A técnica de extração por dispersão da matriz em fase sólida (DMFS), introduzida por Barker et al. ${ }^{6}$ resolveu os problemas da extração de matrizes sólidas, semissólidas e viscosas, ${ }^{7}$ principalmente em análises de amostras biológicas que, devido à complexidade das mesmas, exigem preparo da amostra, sendo esta a etapa mais problemática. A DMFS está baseada em princípios químicos e físicos simples, envolvendo forças aplicadas à amostra por mistura mecânica, para produzir

*e-mail: shgb@uol.com.br rompimento da amostra e interações da mesma com o suporte sólido e com a fase quimicamente ligada (C18) ou com a superfície química de outros suportes sólidos. ${ }^{7}$ A escolha da técnica de extração a ser utilizada deve levar em conta as características: ser simples, rápida, de baixo custo, fornecer extratos relativamente livre de interferentes, proporcionar altas recuperações, boa exatidão e precisão. ${ }^{8}$ A DMFS apresenta vantagens sobre as técnicas convencionais de extração, pois utiliza pequena quantidade de amostra, sem manipulações químicas complicadas, poucas etapas envolvidas e pequeno consumo de solventes orgânicos. ${ }^{9}$

A cromatografia representa o mais relevante conjunto de técnicas analíticas disponíveis atualmente para análise de substâncias químicas, ${ }^{10} \mathrm{em}$ especial a cromatografia gasosa, devido os baixos limites de detecção que podem ser conseguidos com a técnica. ${ }^{11}$ A maioria dos métodos físico-químicos, para a confirmação de resíduos de medicamentos veterinários em alimentos, utiliza a cromatografia e as técnicas espectrométricas, as quais fornecem informações sobre as estruturas químicas dos analitos, sendo recomendadas para a confirmação inequívoca da identidade, evitando com isso a possibilidade de resultados falso-positivos. No que diz respeito às determinações de contaminantes em alimentos, as técnicas cromatográficas de separação destacam-se no âmbito analítico pela reconhecida capacidade de possibilitarem análises qualitativas e quantitativas. ${ }^{12}$

O desenvolvimento de um método analítico, a adaptação ou a implementação de um método conhecido envolve um processo de avaliação que estime sua eficiência na rotina do laboratório, o qual é denominado de validação. ${ }^{13}$ A validação é imprescindível para garantir a confiabilidade dos resultados de um determinado procedimento analítico. ${ }^{12}$ Quando a tomada de decisões for baseada em resultados analíticos, é importante ter alguma indicação quanto à qualidade dos resultados, ou seja, o quanto se pode confiar neles para cada propósito pretendido. ${ }^{14}$ Os parâmetros de validação de métodos analíticos envolvem especificidade/seletividade, função da resposta (gráfico analítico), intervalo de trabalho, linearidade, sensibilidade, exatidão, precisão (repetitividade, precisão intermediária e reprodutividade), limite de detecção (LD), limite de quantificação (LQ) e robustez. ${ }^{13}$

Foi publicada recente revisão bibliográfica sobre os muitos usos da DMFS na extração e análise de compostos de várias classes, envolvendo diferentes matrizes. ${ }^{7}$ Entretanto, não foi registrado nenhum 
estudo envolvendo a matriz plasma, bem como, com os analitos contemplados neste estudo, fipronil e clorfenvinfos.

O objetivo do presente estudo foi desenvolver e validar um método analítico, empregando a dispersão da matriz em fase sólida (DMFS), seguida pela cromatografia gasosa acoplada à espectrometria de massas (GC-MS), na análise de resíduos dos pesticidas clorfenvinfos, fipronil e cipermetrina, os quais são aplicados no rebanho bovino no combate ao carrapato Boophilus microplus, utilizando como matriz de estudo o plasma bovino. Aplicar o método analítico desenvolvido em amostras de plasma de animais submetidos ao tratamento com os pesticidas.

\section{PARTE EXPERIMENTAL}

\section{Materiais e reagentes}

Os padrões analíticos dos pesticidas, clorfenvinfos, fipronil e cipermetrina, foram adquiridos da Sigma Aldrich (Alemanha), com pureza superior a $96,7 \%$, sendo as soluções estoque, concentração de $100 \mathrm{mg} \mathrm{L}^{-1}$, bem como as de trabalho, em concentrações diferenciadas, preparadas em solvente acetonitrila grau HPLC da Mallinckrodt (Pillipsburg, NJ, USA). Octadecylsilano (C18, $50 \mu \mathrm{m})$ e florisil (100200 mesh) foram adquiridos da J. T. Baker (Phillipsburg, NJ, USA) e $\mathrm{Na}_{2} \mathrm{SO}_{4}$ da Synth (Brasil).

As amostras de sangue foram adquiridas de animais procedentes do rebanho bovino da Embrapa Pecuária Sudeste, sendo coletadas em bolsa de transfusão, contendo o anticoagulante citrato de sódio. No laboratório o sangue foi centrifugado, utilizando-se centrífuga marca Fanem (Brasil), por $10 \mathrm{~min}$, a 3000 rpm, separando-se a parte líquida (plasma) da sólida (precipitado).

\section{Preparo da amostra}

Neste estudo foi aplicada a técnica de extração por dispersão da matriz em fase sólida (DMFS), descrita em Lehotay et al. ${ }^{15} \mathrm{e}$ Schenck e Wagner. ${ }^{16}$

Na DMFS 0,25 mL de plasma foi fortificado com a solução de trabalho dos padrões analíticos, permanecendo em repouso por $30 \mathrm{~min}$, de modo que ocorresse a interação entre os analitos e a matriz. Em seguida adicionou-se $1 \mathrm{~g}$ de $\mathrm{C} 18$ e $1 \mathrm{~g}$ de $\mathrm{Na}_{2} \mathrm{SO}_{4}$, homogeneizandose a mistura em almofariz por $2 \mathrm{~min}$, sendo o conteúdo transferido para um cartucho de SPE de polietileno, volume $20 \mathrm{~mL}$, contendo $1 \mathrm{~g}$ de florisil pré-ativado com $5 \mathrm{~mL}$ de acetonitrila. Os pesticidas foram eluídos com $15 \mathrm{~mL}$ de acetonitrila, sendo o eluato rotaevaporado até a secura, em seguida reconstituído com $1 \mathrm{~mL}$ de solvente acetonitrila e injetado no GC-MS.

\section{Análises cromatográficas: GC-MS}

As análises cromatográficas foram feitas utilizando um cromatógrafo a gás, equipado com detector seletivo de massas, marca Shimadzu (Kyoto, Japão), modelo GCMS-QP2010, com coluna capilar de sílica fundida DB-5 (30 m x 0,25 mm x 0,1 $\mu \mathrm{m})$. O pesticida cipermetrina é constituído por oito isômeros ópticos, mas a técnica de separação GC tem a capacidade de distinguir apenas quatro, cis1, cis 2 , trans 1 e trans $2 .{ }^{17}$ As condições analíticas foram: temperatura do injetor, interface e fonte de íons de $250{ }^{\circ} \mathrm{C}$, gás de arraste hélio com vazão na coluna de $0,75 \mathrm{~mL} \mathrm{~min}^{-1}$, injetor no modo sem divisão de fluxo (splitless) e programação de temperatura do forno: $120^{\circ} \mathrm{C}-4$ ${ }^{\circ} \mathrm{C} \min ^{-1}-190{ }^{\circ} \mathrm{C}-32{ }^{\circ} \mathrm{C} \min ^{-1}-270{ }^{\circ} \mathrm{C}$ (4 min), volume de injeção da solução padrão e extrato de $1 \mu \mathrm{L}$. O espectrômetro de massas foi operado no modo MIS (monitoramento de íons selecionados), sendo selecionado três íons para cada analito estudado (Tabela 1), modo de ionização por impacto de elétrons a $70 \mathrm{eV}$.
Tabela 1. Tempo de retenção dos pesticidas analisados, com os respectivos íons monitorados $(\mathrm{m} / \mathrm{z})$, obtidos através do fragmentograma de cada composto

\begin{tabular}{lcccc}
\hline Pesticida & $\begin{array}{c}\text { Tempo retenção } \\
(\mathrm{min})\end{array}$ & \multicolumn{3}{c}{$\mathrm{m} / \mathrm{z}$} \\
\hline Clorfenvinfos & 16,8 & 170 & 267 & 323 \\
Fipronil & 17,1 & 367 & 351 & 420 \\
$\begin{array}{l}\text { Cipermetrina } \\
(4 \text { isômeros })\end{array}$ & 22,$1 ; 22,2 ; 22,3 ; 22,3$ & 163 & 165 & 181 \\
\hline
\end{tabular}

\section{Validação do método analítico}

Para assegurar credibilidade nos dados das análises quantitativas, o método analítico foi validado. Foram considerados os parâmetros: exatidão (recuperação), precisão (coeficiente de variação), coeficiente de determinação, linearidade e limites de detecção e quantificação. ${ }^{10,18} \mathrm{~A}$ exatidão foi calculada através da recuperação, avaliando as concentrações de 0,5; 0,1 e 0,04 $\mathrm{mg} \mathrm{L}^{-1}$ e a linearidade através da injeção dos extratos contendo os analitos em estudo no GC-MS, em triplicata, após aplicar a DMFS, analisando as concentrações de 0,04 ; 0,$1 ; 0,25$ e $0,5 \mathrm{mg} \mathrm{L}^{-1}$, construindo-se curvas analíticas. Calculou-se o limite de detecção (LOD) para cada analito, multiplicando-se o valor médio do ruído, na região do tempo de retenção do composto, por três, ${ }^{18}$ e o limite de quantificação como sendo a menor concentração do composto que pode ser medida com uma precisão especificada, dentro do critério de aceitação do método. ${ }^{19}$

\section{RESULTADOS E DISCUSSÃO}

A Figura 1 apresenta os cromatogramas obtidos ao aplicar a DMFS em amostras de plasma bovino, isento dos princípios ativos (A) e fortificado com os pesticidas na concentração de $0,04 \mathrm{mg} \mathrm{L}^{-1}(\mathrm{~B})$, a qual foi efetiva na extração de clorfenvinfos, fipronil e cipermetrina, bem como, atuou no clean-up da amostra, sendo o cromatograma obtido livre de interferentes na região próxima ao tempo de retenção dos compostos analisados.

Segundo Husain et al., ${ }^{20}$ a técnica de extração por dispersão da matriz em fase sólida (DMFS) tem sido amplamente aplicada na extração de drogas veterinárias, herbicidas, pesticidas e outros poluentes presentes em matrizes complexas, como tecidos animais, frutas e vegetais. Na extração as interações entre os componentes individuais da amostra e o analito de interesse envolvem o analito com o suporte sólido, o analito com a fase orgânica ligada, o analito com a matriz dispersa, a matriz com o suporte sólido, a matriz com fase orgânica ligada e todos estes componentes interagindo com o solvente de eluição, interações estas que ocorrem simultaneamente. Assim, a escolha do suporte-adsorvente recai na polaridade do analito e na natureza da matriz, de modo que o isolamento de analitos mais polares é realizado com suportes sólidos polares e o de analitos menos polares com suportes menos polares, sendo que a quantidade e a porcentagem também afetam os resultados. .,21,22 $^{2}$

A validação de um método analítico na área de resíduos de pesticidas é realizada para garantir que a mesma seja exata, específica e reprodutível, e envolve a interação entre o princípio ativo e a matriz durante os procedimentos analíticos, que resulta na quantificação por métodos instrumentais. ${ }^{23}$ A Tabela 2 ilustra os resultados obtidos na validação do método proposto. Os valores de recuperação obtidos para clorfenvinfos, fipronil e cipermetrina, extraídos da matriz plasma, avaliando-se as concentrações de 0,5; 0,1 e 0,04 $\mathrm{mg} \mathrm{L}^{-1}$, variaram de 70 a $126 \%$, com coeficientes de variação $(\mathrm{CV})$ compreendidos entre 2 e $8 \%$. Esses valores estão dentro da faixa de aceite estabelecida 
pelo EPA, a qual varia de 70 a $130 \%$, com CV inferior a $30 \%,{ }^{24}$ sendo adequados às análises nos níveis estudados.
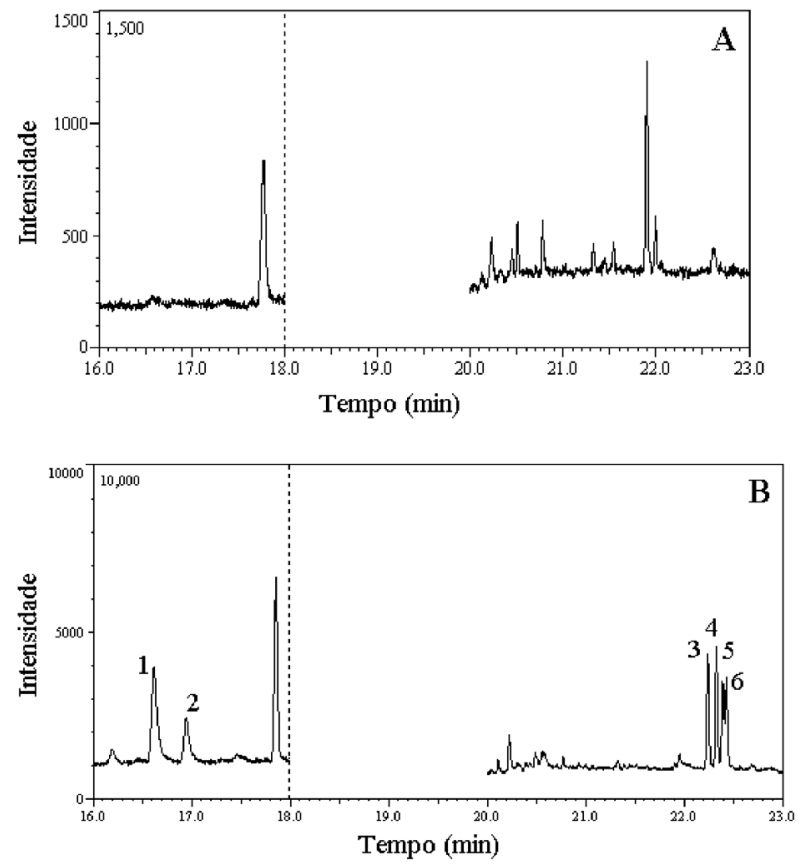

Figura 1. Cromatogramas dos extratos após aplicar o método analítico, dispersão da matriz em fase sólida seguida pela cromatografia gasosa acoplada à espectrometria de massas, em amostras de plasma bovino, na ausência dos pesticidas (A) e fortificação com os analitos na concentração de 0,04 $\mathrm{mg}^{\mathrm{L}^{-1}}$ (B). MS operando no modo MIS. Coluna capilar de sílica fundida DB-5 (30 $m \times 0,25 \mathrm{~mm} \times 0,1 \mu \mathrm{m})$. Programação de temperatura do forno: $120^{\circ} \mathrm{C}-4^{\circ} \mathrm{C}$ $\mathrm{min}^{-1}-190^{\circ} \mathrm{C}-32{ }^{\circ} \mathrm{C} \mathrm{min}^{-1}-270^{\circ} \mathrm{C}(4 \mathrm{~min}) .1=$ clorfenvinfos; 2 = fipronil; $3,4,5,6=$ isômeros da cipermetrina

Tabela 2. Valores de recuperação e coeficientes de variação (CV), obtidos ao aplicar a dispersão da matriz em fase sólida (DMFS) seguida pela cromatografia gasosa acoplada à espectrometria de massas (GCMS), na análise de clorfenvinfos, fipronil e cipermetrina, em amostras de plasma, avaliando as concentrações de 0,$5 ; 0,1$ e $0,04 \mathrm{mg} \mathrm{L}^{-1}$

\begin{tabular}{lccc}
\hline Pesticida & \multicolumn{3}{c}{ Recuperação (\%) $\pm \mathrm{CV}(\%)$} \\
& $0,5 \mathrm{mg} \mathrm{L}^{-1}$ & $0,10 \mathrm{mg} \mathrm{L}^{-1}$ & $0,04 \mathrm{mg} \mathrm{L}^{-1}$ \\
\hline Clorfenvinfos & $73 \pm 2$ & $120 \pm 5$ & $85 \pm 8$ \\
Fipronil & $70 \pm 3$ & $93 \pm 4$ & $126 \pm 5$ \\
Cipermetrina & $73 \pm 2$ & $116 \pm 4$ & $104 \pm 5$ \\
\hline
\end{tabular}

Na determinação de resíduos de medicamentos veterinários em alimentos, os métodos analíticos mais apropriados são os cromatográficos associados à espectrometria de massas, mas é importante incluir no protocolo o parâmetro efeito matriz. A presença de componentes coextraídos da matriz pode causar sérios problemas nas análises cromatográficas, incluindo imprecisão na quantificação, decréscimo na robustez do método, baixa detectabilidade dos analitos e, mesmo, a obtenção de resultados falso positivo ou negativo. ${ }^{25}$ Ao injetar a amostra real, componentes da matriz tendem a bloquear os sítios ativos, principalmente os grupos silanois livres, no injetor ou coluna cromatográfica, onde ocorre a adsorção ou degradação dos analitos. ${ }^{26}$ Este fenômeno resulta em um maior sinal do analito na solução contendo a matriz do que na livre da matriz. Outro problema associado com a injeção da matriz envolve a gradual acumulação de componentes não voláteis no sistema GC, resultando na formação de novos sítios ativos, e gradual diminuição/redução na resposta do analito. Portanto, diante da complexidade da matriz de estudo, o plasma bovino, e visando atender à legislação, com valores de recuperação e coeficientes de variação dentro da faixa de aceite, constantes limpezas do liner e coluna cromatográfica foram necessárias durante o desenvolvimento e aplicação do método proposto.

Os limites de detecção (LOD) e quantificação (LOQ) foram 0,01 e $0,04 \mathrm{mg} \mathrm{L}^{-1}$ para clorfenvinfos e fipronil, respectivamente, e 0,005 e $0,02 \mathrm{mg} \mathrm{L}^{-1}$ para cipermetrina, respectivamente. A linearidade foi calculada construindo curvas de calibração externa com os analitos em estudo, depois de extraídos do plasma bovino, obtendo os respectivos coeficientes de determinação e equações de regressão, variando as concentrações de 0,04 a $0,50 \mathrm{mg} \mathrm{L}^{-1}$ (Figura 2). O método desenvolvido, dispersão da matriz em fase sólida seguido pela cromatografia gasosa acoplada à espectrometria de massas, mostrou-se linear nas concentrações analíticas analisadas, com coeficientes de determinação para os três analitos superiores a 0,96 , demonstrando que o detector de massas responde bem às concentrações injetadas.

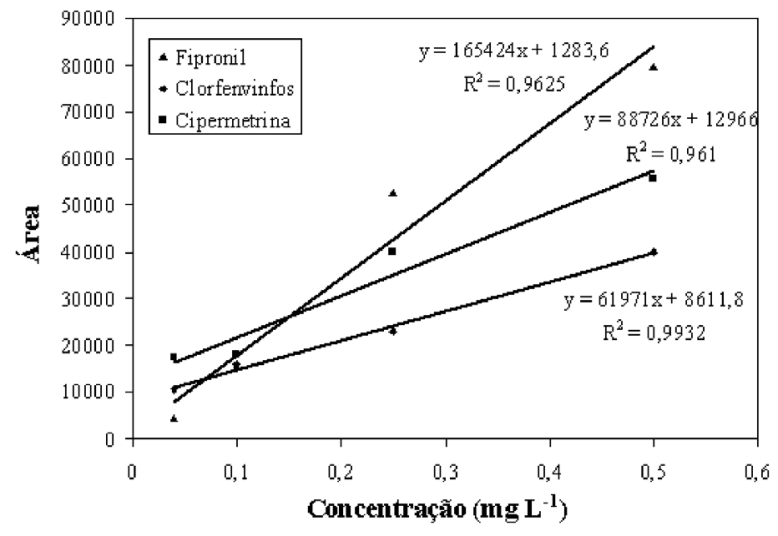

Figura 2. Curvas analíticas para os pesticidas cipermetrina, clorfenvinfos e fipronil, nas concentrações de 0,04; 0,1;0,25 e 0,5 $\mathrm{mg} \mathrm{L}^{-1}$

Um aspecto importante a ser considerado em se tratando de métodos destinados à quantificação de resíduos de medicamentos veterinários em alimentos, é o fato de que apenas garantir a confiabilidade analítica das medidas não é suficiente. Na validação deve ser levado em consideração o limite máximo de resíduo (LMR) da substância alvo no alimento, no intuito de garantir que o método seja adequado para o objetivo proposto, ou seja, no controle da qualidade de alimentos visando a inocuidade alimentar. ${ }^{12}$ Como não existe um valor estabelecido de LMR para a matriz plasma bovino, consideraram-se os LMRs estabelecidos para a cipermetrina em leite e carne, $0,1 \mathrm{e}$ $0,05 \mathrm{mg} \mathrm{kg}^{-1}$, respectivamente, ${ }^{27}$ valores esses superiores aos LOQs determinados no estudo, não existindo valores definidos de LMRs para clorfenvinfos e fipronil.

Após desenvolvido, otimizado e validado, o método analítico proposto foi aplicado em amostras de plasma de bovinos, procedentes de animais submetidos ao tratamento com os pesticidas, sendo possível identificar a presença do analito cipermetrina 6 e 24 h após a aplicação do composto (Figura 3). Considerando os valores de área obtidos e aplicando a equação da reta, referente à curva de calibração da cipermetrina, as concentrações detectadas foram inferiores ao limite de quantificação do método.

\section{CONCLUSÃO}

O método proposto mostrou-se sensível, preciso e reprodutível para analisar resíduos dos pesticidas clorfenvinfos, fipronil e ciper- 


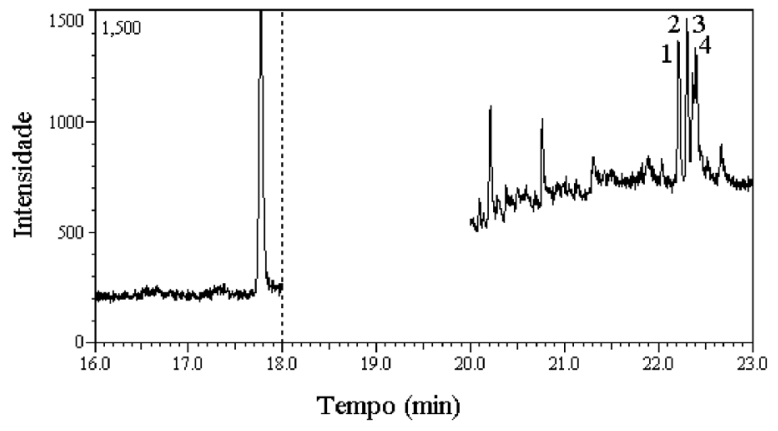

Figura 3. Cromatograma do extrato após aplicar a DMFS e posterior análise por GC-MS, modo SIM, em amostra de plasma procedente de animal submetido ao tratamento com os pesticidas. Programação de temperatura do forno: $120^{\circ} \mathrm{C}-4^{\circ} \mathrm{C} \mathrm{min}{ }^{-1}-190^{\circ} \mathrm{C}-32^{\circ} \mathrm{C} \mathrm{min}{ }^{-1}-270^{\circ} \mathrm{C}(4 \mathrm{~min})$. Coluna DB-5 $(30 \mathrm{~m}$ x 0,25 mm $\times 0,1 \mu \mathrm{m}) .1,2,3,4=$ isômeros da cipermetrina

metrina em plasma bovino, uma vez que estes foram detectados a baixas concentrações: $0,005 \mathrm{mg} \mathrm{L}^{-1}$ para cipermetrina e $0,01 \mathrm{mg} \mathrm{L}^{-1}$ para clorfenvinfos e fipronil, com valores de recuperação e precisão dentro do intervalo de aceite estabelecido pelo EPA. A técnica de extração aplicada, dispersão da matriz em fase sólida (DMFS), é simples, rápida, eficiente e de baixo custo, com reduzido consumo de solventes orgânicos. Analisando amostras de plasma de bovinos submetidos ao tratamento com os pesticidas, foi possível detectar a presença do analito cipermetrina, com concentrações detectadas inferiores ao limite de quantificação do método. O método analítico pode ser utilizado como um protocolo para investigar a presença de resíduos de clorfenvinfos, fipronil e cipermetrina, em amostras de plasma de origem bovina.

\section{AGRADECIMENTOS}

À FAPESP pelo suporte financeiro à pesquisa (auxílios $\mathrm{n}^{\circ}$ 04/11736-9; 05/53188-0; 07/54669-8.

\section{REFERÊNCIAS}

1. http://www.incqs.fiocruz.br/programas_institucionais/alimentos/pamvet. htm, acessada em Julho 2009.

2. http://www.abrappa.org.br/eventos/simp_alim_3, acessada em em Julho 2009.
3. Luchiari, F.; II SIMBOI - Simpósio sobre desafios e novas tecnologias na bovinicultura de corte, Brasília, Brasil, 2006.

4. Lobato, V.; Tese de Doutorado, Universidade Estadual de Campinas, Brasil, 2001.

5. Sanches, S. M.; Silva, C. H. T. P.; Campos, S. X..; Vieira, E. M.; Pesticidas: Revista de Ecotoxicologia e Meio Ambiente 2003, 13, 53.

6. Barker, S. A.; Long, A. R.; Short, C. R.; J. Chromatogr., A 1989, 475, 353.

7. Barker, S. A.; J. Biochem. Biophys. Methods 2007, 70, 151.

8. EPA (Environmental Protection Agency); Pesticides - The EPA and Food Security, 2004.

9. Dórea, H. S.; Lopes, W. G.; Quim. Nova 2004, 27, 892.

10. Lanças, F. M.; Validação de métodos cromatográficos de análise, Rima: São Carlos, 2004.

11. Collins, C. H.; Braga, G. L.; Bonato, P. S.; Fundamentos de Cromatografia, UNICAMP Editora: Campinas, 2006.

12. Paschoal, J. A. R.; Rath, S.; Airoldi, F. P. S.; Reyes, F. G. R.; Quim. Nova 2008, 31, 1190

13. Brito, N. M.; De Amarante Junior, O. P.; Polese, L.; Ribeiro, M. L.; Pesticidas: Revista de Ecotoxicologia e Meio Ambiente 2003, 13, 129.

14. Ellison, S. L. R.; Rosslein, M.; Willians, A.; Guia EURACHEM/CITAC: Determinando a incerteza na medição analítica, QUAM: 2002 - Versão Brasileira.

15. Lehotay, S. J.; Mastovska, K.; Yun, S. J.; J. AOAC Int. 2005, 88, 630.

16. Schenck, F. J.; Wagner, R.; Food Addit. Contam. 1995, 12, 535.

17. Sassine, A.; Moura, S.; Léo, V. M.; Bustillos, O. V.; J. Anal. Toxicol. 2004, 28, 238.

18. Leite, F.; Validação em análise química, Átomo Ltda: Campinas, 1996.

19. Cass, Q. B.; Degani, A. L. G.; Desenvolvimento de métodos por HPLC. Fundamentos, estratégias e validação, Editora da UFSCar: São Carlos, 2001.

20. Husain, S. W.; Kiarostami, V.; Morrovati, M.; Tagebakhsh, M. R.; Acta Chromatogr. 2003, 13, 208.

21. Barker, S. A.; LCGC North Am. 1998, S, 537.

22. Barker, S. A.; J. Chromatogr., A 2000, 880, 63.

23. Vieira, E.; Lichtig, J.; Arq. Inst. Biol. 2004, 71, 1.

24. Tolosa, I.; Readman, J. W.; Mee, L. D.; J. Chromatogr., A 1996, 725, 93.

25. Mastovská, K.; Lehotay, S. J.; Anastassiades, M.; Anal. Chem. 2005, 77, 8129.

26. Schenck, F. J.; Lehotay, S. J.; J. Chromatogr., A 2000, 868, 51.

27. http://www.who.int/pcs/food/jecfa/jecfa.htm, acessada em Julho 2009. 